\title{
Overestimation of subfoveal choroidal thickness by measurement based on horizontally compressed optical coherence tomography images
}

\author{
Jae Hui Kim • Se Woong Kang • Jae Ryung Kim
}

Received: 23 August 2013 / Accepted: 26 August 2013 / Published online: 15 September 2013

(C) Springer-Verlag Berlin Heidelberg 2013

Dear Editor,

We would like to thank you for the valuable comment of Tan and colleagues that pointed out the possible influencing factors in optical coherence tomography (OCT) analysis as well as the difference between high-speed mode and high-resolution mode.

In our study [1], all the OCT scans were performed using the high-speed mode. As the comment of Tan and colleagues, there is an obvious difference in degree of horizontal image compression in 1:1 pixel image between high-speed mode and highresolution mode. Because the high-speed mode shows a greater degree of horizontal compression in a 1:1 pixel image, the overestimation of subfoveal choroidal thickness measured based on the 1:1 pixel image may be exaggerated when using the highspeed mode than in using the high-resolution mode. It was our inadvertent inattentiveness to confuse the high-resolution image set by $1: 1$ pixel with the image set by $1: 1$ micron that was presented in their previous study [2]. However, it should be emphasized that a certain degree of horizontal compression, as shown in Figure in the letter of Tan and colleagues, is inevitable even with the high-resolution image in 1:1 pixel display setting.

\author{
J. H. Kim \\ Department of Ophthalmology, Kim's Eye Hospital, \\ Konyang University College of Medicine, \\ Seoul, South Korea \\ S. W. Kang $(\bowtie) \cdot J$. R. Kim \\ Department of Ophthalmology, Samsung Medical Center, \\ Sungkyunkwan University School of Medicine, \\ \#50 Irwon-dong, Kangnam-gu, \\ Seoul 135-710, South Korea \\ e-mail: swkang@skku.edu \\ S. W. Kang \\ e-mail: kangsewoong@gmail.com
}

Thus, the primary conclusion of our study, which includes overestimation of choroidal thickness in horizontally compressed images, may also be valid in this case.

We also agree with the opinion of Tan and colleagues that the degree of magnification may have an influence on determining the boundary of layers. To the best of our knowledge, the optimal degree of magnification that allows the most accurate determination of boundary has not yet been investigated. In our experience, it was difficult to determine the accurate boundary of layers in a very high degree of magnification as well as a low degree of magnification. Thus, a moderate degree of magnification (400\%) was used in our study [1]. We hope further studies may reveal the optimal degree of magnification in various OCT devices.

In summary, the mode of display setting of an OCT image (1:1 pixel vs. 1:1 micron), the mode of scanning (high resolution vs. high speed), and the degree of image magnification should be considered for the accurate measurement of thickness in retino-choroidal layers.

Meeting presentation None.

Financial support None.

\section{References}

1. Kim JH, Kang SW, Ha HS, Kim SJ, Kim JR (2013) Overestimation of subfoveal choroidal thickness by measurement based on horizontally compressed optical coherence tomography images. Graefes Arch Clin Exp Ophthalmol 251:1091-1096

2. Tan CS, Ouyang Y, Ruiz H, Sadda SR (2012) Diurnal variation of choroidal thickness in normal, healthy subjects measured by spectral domain optical coherence tomography. Invest Ophthalmol Vis Sci 53:261-266 